\title{
NEW RESULTS FOR THE MASS-INERTIAL PARAMETERS OF THE HUMAN BODY BASED ON 3D MATHEMATICAL MODELLING
}

\author{
Gergana Nikolova*, Daniel Dantchev, Mihail Tsveov \\ Institute of Mechanics, Bulgarian Academy of Sciences, \\ Acad. G. Bonchev. Str., Bl. 4, 1113 Sofia, Bulgaria
}

[Received: 17 April 2020. Accepted: 05 October 2020]

doi: 10.7546/JTAM.50.20.04.05

\begin{abstract}
Geometric modelling is one of the methods for determination of the mass-inertial parameters of the different segments of the body, as well as of the body as a whole. The current article presents new 20-segment 3D mathematical model of the human body, generated in a computer environment, allowing the mass-inertial parameters of all body segments to be calculated. Some data obtained from it for all segments of the body are reported. We also present a brief review of some of our results related to $i$ ) a new type of mathematical modelling of the thigh of the human body; ii) investigation of mass - inertial characteristics in basic body position as selected by NASA. Next, we outline some further improvements and future developments of the models under consideration. Let us note that the proposed models are oriented towards applications in medicine (orthopaedics and traumatology), rehabilitation robotics, computer simulations, sports and areas such as ergonomics, forensics and more.
\end{abstract}

KEY WORDS: biomechanics, mass-inertial parameters, 3D mathematical modelling, CAD design.

\section{INTRODUCTION}

Let us start with the obvious observation that the knowledge of geometrical and the mass-inertial characteristics of the human body is needed when studying its movement, or ability to move. Naturally, this is true both for the kinematic as well as of dynamic analysis of the motion. Nowadays there are new modern topics in that direction connected to movements, say, in microgravity or, say, design of wearable and rehabilitation robots. It shall be no surprise, however, that studies of human motion have started long ago - according to some sources already at about 1000 B.C. An interesting historic perspective of these investigations in the early years can be found in Ref. [1]. In addition, a curious reader can also see Refs. [2,3]. For a general overview on the topic, without pretending to be exhaustive, the reader can consult, e.g., Refs. [4-7]. The necessity to know the characteristics of the human body is def-

\footnotetext{
*Corresponding author e-mail: gergana1973@gmail.com
} 
initely needed and has been object of intensive studies in medicine (orthopaedics and traumatology, especially gait analysis and orthotics) [8-13], sport (both for driving highest possible results and for different matters related to athletic injuries) [14-18], criminology [19,20], ergonomics [21], for devising of assistive devices [22], etc.

Accompanying the emergence of new areas of research related to human massinertial characteristics, one observed an evolution in the methods which have been implemented, or are still in use, for obtaining the information needed. In the 1960's and 1970 's, several studies reported anthropometric and mass-inertial parameters for the human body segments of adult male cadavers [23, 24,26]. Later on, different methods for investigation of body segment parameters of the living males and females have been used: immersion and cast method [26], gamma mass scanning [27, 28], magnetic resonance imaging [29], computerized tomography [30], geometrical modelling [31-33], etc. In the present study, we use an approach based on 3D geometrical modelling.

When one represents via a mathematical model the human body, or a combination of its segments, one should successively solve specific problems, related to:

1. Appropriate body decomposition - descriptions of the anthropometric points outlining segments and relevant characteristic lengths.

2. Generation of a proper 3D model that includes the decision which segment of the body shall be modelled with what geometrical body.

3. Analytical determination of the characteristics of the segments of interest such as mass, the centre of mass, moments of inertia using the mathematical properties of the 3D bodies involved.

4. Determining the parameters as the lengths of the respective segments using data from anthropological measurements, as well as from measurements of the density of the segments.

5. Computer realization of the $3 \mathrm{D}$ model with the data determined in the previous point.

6. Verification of the computer-generated model by comparing the data obtained from the determination of the human body mass properties, using analytically derived results with those obtained based on computer realization.

7. Determination of the characteristics of interest of a particular part of the body, or the body as a whole, using the computer realization for, say, special positions and movements for which the analytical calculation would be time-consuming, cumbersome, or difficult. 
In the present article, we will use the aforementioned recipe to study the massinertial properties of the human body via a combination of mathematical and computer modelling. We will present a 20 -segment 3D mathematical model of the human body which will be generated in a computer environment that allows not only the mass-inertial parameters of the single body segments to be calculated, which we have done also analytically, but these characteristics for the whole body in any position of interest. We will devise a new type of modelling of the human thigh that solves the long standing problem in the mathematical modelling of the human body of the continuous tailoring of the thigh with the torso keeping the corresponding anthropometric angles as given in the literature. We will report results for the mass-inertial characteristics of the human body of the average male and female in several body positions as classified by NASA as envisaged for space exploration. Finally, we will outline the perspectives for further improvement of our studies.

\section{BASic FACTS For the Mathematical Model}

Let us start with the presentation of the main mathematical model we are going to use for studies of the mass-inertial parameters of the human body. It consists of 20 segments, see Fig. 1a: head + neck, the upper part of the torso, the middle part of the torso, the lower part of the torso, three parts of the thigh, shank, foot, upper arm, lower arm, and hand. All segments are assumed to be relatively simple geometrical bodies.

We suppose full-body symmetry to the sagittal plane, i.e., complete left - right symmetry. Details of the exact dimensions, decomposition of the body according to anthropometric points for all segments except those of the thigh, which will be given in the following paragraph, can be found in [34]. The geometrical data needed for the determination of all lengths required to make the model specific is taken from a detailed representative anthropological investigation of the Bulgarian population [35] performed during the period 1989 - 1993. A total of 2435 males and 2855 females were measured. We take the average values found in the above investigation and design a model, which represents the so defined "average" Bulgarian male, or female. The segments are modelled through 3D geometrical forms similar to those in [31] but with the following modifications: (1) the torso is subdivided into three instead of two parts; (2) the torso upper part is approximated using a right reverted elliptical cone, while it is an elliptical cylinder in [31]; (3) according to [4], we specify both middle and lower torso modelled as an elliptical cylinder and an elliptical cylinder +reverted elliptical cone, respectively; in [31] these two segments are grouped and modelled as an elliptical cylinder. Take notice that the torso lower part is defined here exactly as in [4]: it extends from omphalion to iliospinale, with a plane passing through the 

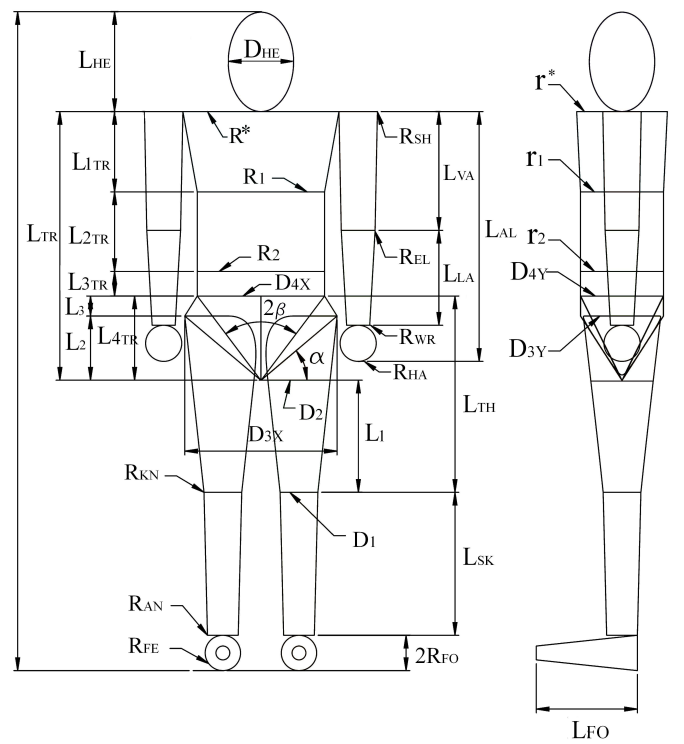

(a) Outline of the mathematical model of the human body.

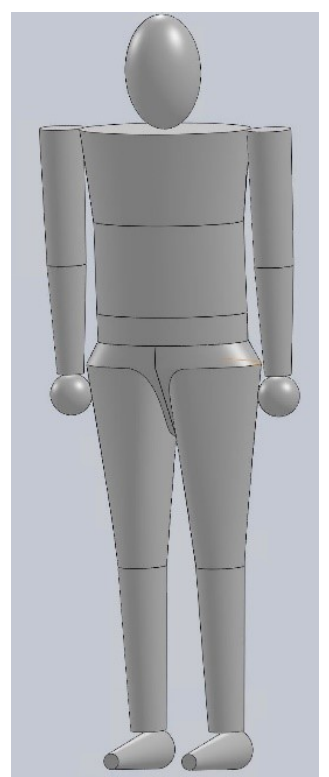

(b) The computer realization of the model in CAD system.

Fig. 1: Visualisation of the model of the human body.

iliospinales and concluding an angle of $37^{\circ}$ with the sagittal plane. We consider the thigh consisting three elements - see below for details; the upper arm, lower arm and shank are assumed to be cone frustums and the hand is modelled as a sphere. Once the geometrical parameters of the segments are determined, one can analytically obtain all the characteristics of interest, such as volume, mass and moments of inertia. Then the so developed 20 segmental mathematical model of the human body of the average Bulgarian man has been generated using CAD/CAM system SolidWorks, see Fig. 1b. The SolidWorks model analysis of each segment yields the following data: a) volume and mass for given density; $b$ ) position of the mass centre; $c$ ) inertial tensor with respect to a reference coordinate frame; $d$ ) inertial tensor with respect to mass centre (radius of gyration); $e$ ) principal inertial moments and principal axis position; g) rotation ellipsoid.

A comparison of the results found by using the software with those from analytical estimations confirms the correctness of the generated computer model. This step is important and allows us to use the computer model to determine all parameters 
Table 1: Moments of inertia of the body segments through the centre of mass $\left[\mathrm{kg} \cdot \mathrm{cm}^{2}\right]$.

\begin{tabular}{|c|c|c|c|c|c|c|c|c|c|}
\hline Segment & \multicolumn{3}{|c|}{ Zatsiorsky [4] } & \multicolumn{3}{|c|}{ Shan, Bohn [37] } & \multicolumn{3}{|c|}{ Our data } \\
\hline Head & 293.9 & 272.1 & 202.4 & 354.0 & 297.3 & 197.6 & 260.5 & 260.5 & 150.0 \\
\hline Upper torso & 705.2 & 1725.6 & 1454.5 & 884.0 & 1868.3 & 1848.3 & 997.0 & 1530.1 & 1421.0 \\
\hline Middle torso & 819.1 & 1280.8 & 1203.1 & 546.4 & 1114.4 & 1231.4 & 784.0 & 1254.3 & 1235.0 \\
\hline Lower torso & 525.0 & 656.8 & 592.4 & 860.4 & 1169.0 & 1167.0 & 430.2 & 693.3 & 693.3 \\
\hline Upper arm & 114.4 & 127.3 & 38.9 & 108.8 & 103.8 & 28.4 & 191.7 & 191.7 & 25.1 \\
\hline Lower arm & 60.2 & 64.7 & 12.6 & 49.8 & 54.6 & 7.3 & 62.2 & 62.2 & 8.6 \\
\hline Hand & 8.8 & 13.2 & 5.4 & 5.7 & 4.5 & 2.2 & 4.7 & 4.7 & 4.7 \\
\hline Thigh & 1999.4 & 1997.8 & 413.4 & 1872.6 & 1879.9 & 420.6 & 1824.8 & 1771.2 & 415.5 \\
\hline Shank & 371.0 & 385.0 & 64.6 & 357.3 & 408.9 & 88.3 & 341.2 & 341.2 & 34.0 \\
\hline Foot & 40.0 & 44.4 & 10.3 & 24.2 & 3.6 & 24.5 & 49.9 & 49.9 & 6.6 \\
\hline
\end{tabular}

of interest of the whole body and for any posture of interest. In Table 1 the model results for the principal moments of inertia calculated in SolidWorks media and experimental data published in the literature are compared. We observe that a good agreement is established. Here for each segment a system of axes with an origin at the segment mass centre is defined. The axes coincide with the approximate body axes: the frontal $(x)$, the saggital $(y)$ and the longitudinal $(z)$ ones. Due to the $x-y$ symmetry assumed for the upper arm, lower arm, hand, shank and foot modelling, the principal inertial moments $I_{X X}$ and $I_{Y Y}$ are identical, except for the thigh. Thigh data as a whole are given, due to the impossibility of comparing our three parts of the thigh with the literature - till now no such data have been reported in the published literature.

\section{Modelling Of THE THIGH}

For studying via mathematical modelling the movements of the human body, surely an accurate modelling of the individual segments of the body is required. One specific problem in that respect that till recently did lack a satisfactory solution is about modelling the thigh-torso connection. The point is that one normally models the torso and the thigh with relatively simple geometrical bodies that do not tailor continuously into each other. Furthermore, one naturally shall also take into account the particular geometric properties describing the connections of both. For example, it shall reflect 
the fact that the thigh is dissected from the torso with a plane passing through the anterior superior iliac spine at an angle of $37^{\circ}$ to the midsagittal plane [4]. Finally, the modelling shall be done in such a way that the mass of the torso as well as of the thigh is reproduced as closely as possible. Recently we have attacked these problems in Ref. [36]. Here we outline, very briefly, some of the results of this article.

For any further details concerning the modelling of the thigh, the analytical expressions describing the centre of mass, volume, etc., of its segments, the reader is directed to Ref. [36].

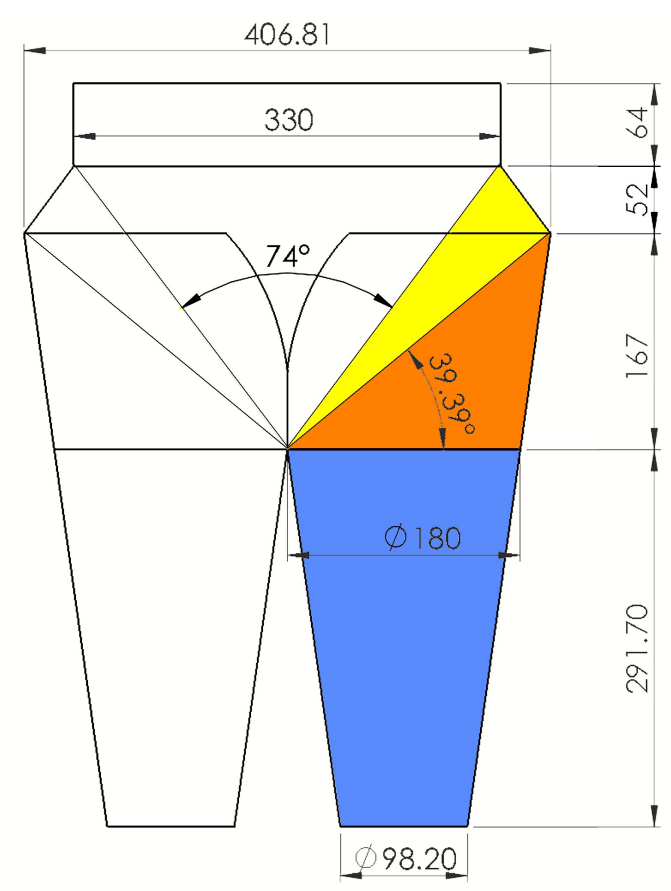

(a) Outline of the mathematical model of the thigh.

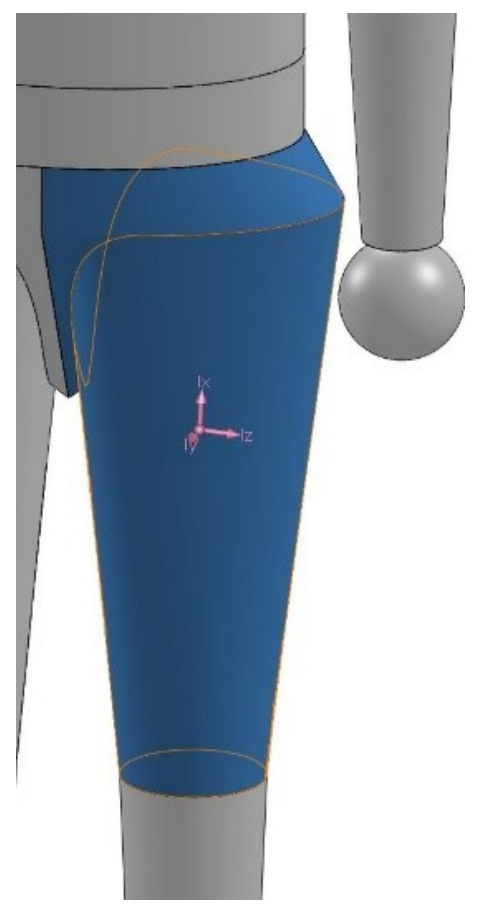

(b) Outline of the CAD model of the thigh.

Fig. 2: Visualization of the model of the thigh. More detailed view on the three segments of the thigh we introduced can be gained from Fig. 3 . 


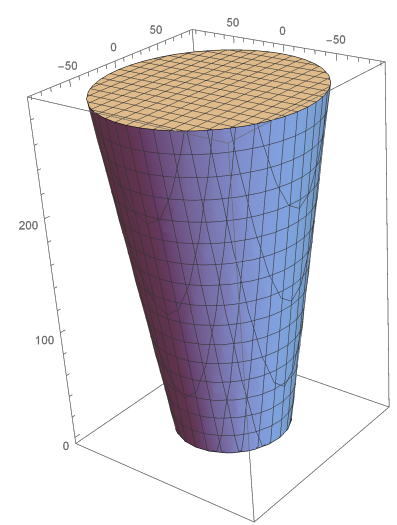

(a) The geometrical body modelling the lower part of the thigh.

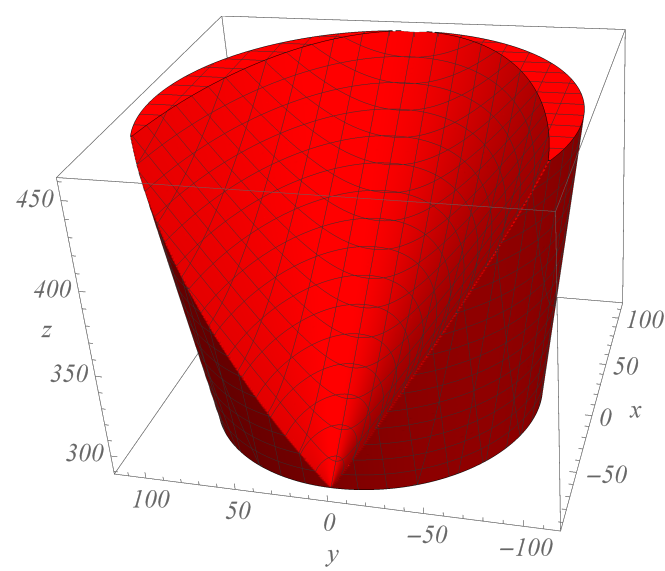

(b) The geometrical body modelling the middle part of the thigh.

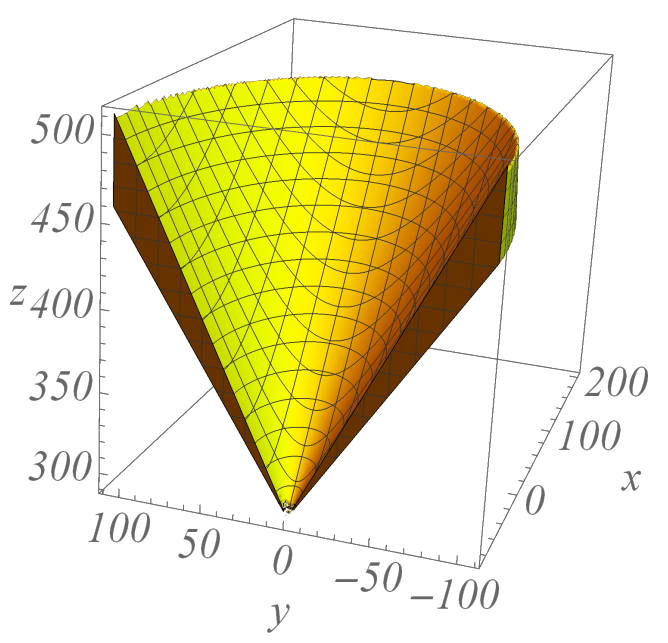

(c) The geometrical body modelling the upper part of the thigh.

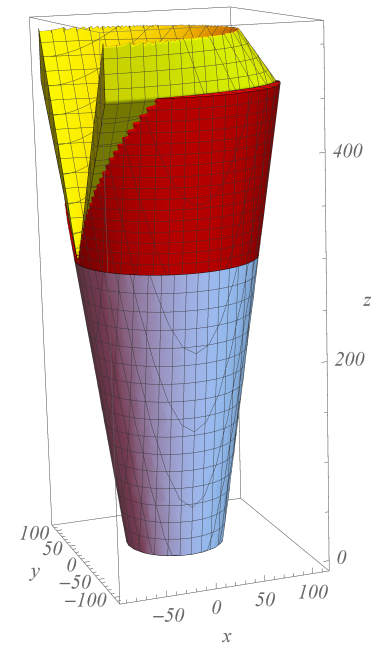

(d) The three segments of the thigh combined.

Fig. 3: More detailed view on the three segments of the thigh. The measures are in $\mathrm{mm}$ and reflect the sizes pertinent to the average Bulgarian male. 


\section{Mass - INERTIAL Characteristics In BASIC Body Position AS SELECTED BY NASA}

As stated in the Introduction, one of the modern topics of research is the specifics related to movement of humans in microgravity. NASA [38] has picked-up eight principal body position of interest and determined in detail the mass-inertial characteristics in these positions for male astronauts. The eight positions are visualized in Fig. 4. Our studies were based on a 16-segmental biomechanical mathematical model of the Bulgarian man and woman originally proposed in [34], that has been also generated within SolidWorks environment. We verified the computer results by comparing its results with the ones for the corresponding segments of the body for which we have derived analytical expression. For example, the inertial moment $I_{\mathrm{XX}}$ for a frustum of an elliptic cone is given by the following equation:

$$
\text { (1) } \begin{aligned}
I_{X X}=\frac{\pi}{240} h \rho\{ & 4 h^{2}\left[r_{1}\left(2 R_{1}+3 R_{2}\right)+3 r_{2}\left(R_{1}+4 R_{2}\right)\right] \\
+3\left[r _ { 1 } ^ { 3 } \left(4 R_{1}+\right.\right. & \left.R_{2}\right)+r_{1}^{2} r_{2}\left(3 R_{1}+2 R_{2}\right) \\
& \left.\left.+r_{1} r_{2}^{2}\left(2 R_{1}+3 R_{2}\right)+r_{2}^{3}\left(R_{1}+4 R_{2}\right)\right]\right\}
\end{aligned}
$$

where $h$ is the length of the segment, $\rho$ its density, $r_{1}$ and $r_{2}$ are the semi-axes at the bottom and $R_{1}$ and $R_{2}$ at the top of the frustum of cone. Let us stress that we did not

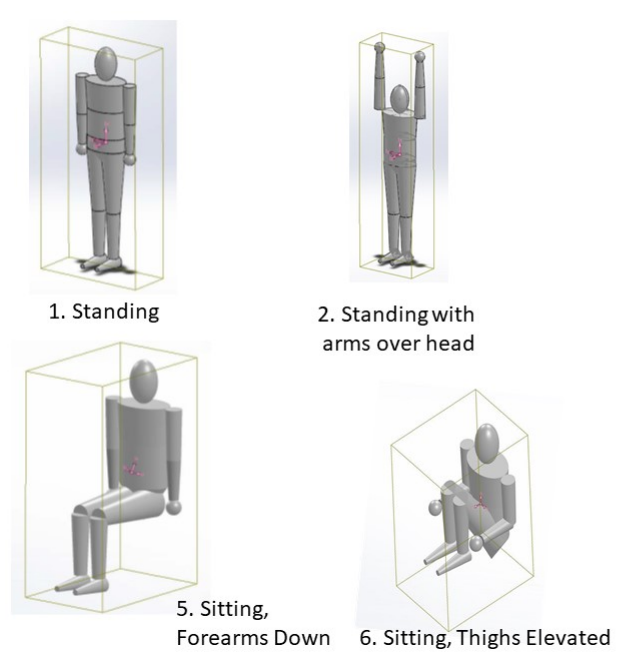

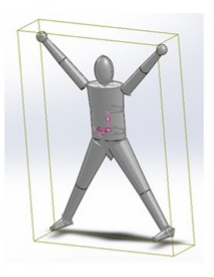

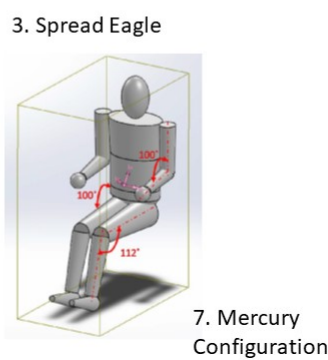

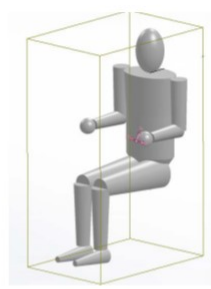

4. Sitting

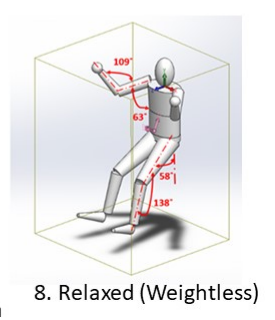

Fig. 4: The eight principal positions of interest for NASA. 
only rely on the actual anthropometric data (say, $h, R_{1}, R_{2}, r_{1}$ and $r_{2}$ ), but also on other important needed parameters, like the density $\rho$ of the corresponding segments taken from the experimental findings. For each position of the body we used a system of axes with origin at the anthropometric point vertex of the head. The axes coincide with the approximate body axes: the frontal (z), the sagittal (y), and the longitudinal (x) ones.

It has been of interest for us to clarify to which extent one can reproduce the NASA's experimental data via mathematical modelling and to which extent the results for average astronaut differ from those for an average Bulgarian male. It turns out [39-41] that for males the results are quite close to those of the astronauts. Inspired by that, we did the corresponding studies also for females, for which NASA

Table 2: Characteristics of the female human body in standing position. Reported are the principal moments of inertia, the average height and mass of the women studied. The centre of mass is measured from the anthropometric point vertex of the head.

\begin{tabular}{lccccc}
\hline \hline Characteristic & \multicolumn{3}{c}{ Young et al., Ref. [44] } & Abraham et al., Ref. [45] & Our data \\
& Min & Max & Mean & & \\
\hline$I_{\mathrm{X} X}\left[\mathrm{~kg} \cdot \mathrm{cm}^{2} \times 10^{3}\right]$ & 5.8 & 24.0 & 11.6 & - & 6.7 \\
$I_{\mathrm{Y} Y}\left[\mathrm{~kg} \cdot \mathrm{cm}^{2} \times 10^{3}\right]$ & 49.1 & 135.0 & 85.0 & - & 78.3 \\
$I_{\mathrm{ZZ}}\left[\mathrm{kg} \cdot \mathrm{cm}^{2} \times 10^{3}\right]$ & 53.0 & 135.0 & 91.9 & - & 81.9 \\
Stature $[\mathrm{cm}]$ & - & - & 161.20 & 162.60 & 160.51 \\
Mass $[\mathrm{kg}]$ & - & - & 63.90 & 64.64 & 60.65 \\
Center of mass $[\mathrm{cm}]$ & - & - & - & - & 67.3 \\
\hline \hline
\end{tabular}

Table 3: Characteristics of the male human body in standing position. Reported are the principal moments of inertia, the average height and mass of the men studied. The centre of mass is measured from the anthropometric point vertex of the head. Moments of inertia of the body segments through the centre of mass $\left[\mathrm{kg} \cdot \mathrm{cm}^{2}\right] \times 10^{3}$.

\begin{tabular}{lccccccc}
\hline \hline Characteristic & $\begin{array}{l}\text { NASA } \\
\text { Ref. [38] }\end{array}$ & \multicolumn{2}{c}{$\begin{array}{c}\text { Chandler et al. } \\
\text { Ref. [26] }\end{array}$} & $\begin{array}{c}\text { Santschi } \\
\text { Ref. [46] }\end{array}$ & $\begin{array}{c}\text { Hanavan } \\
\text { Ref. [31] }\end{array}$ & Our data \\
\hline & $50 \%$ & $95 \%$ & & & $50 \%$ & $95 \%$ & \\
$I_{\mathrm{X} X}$ & 14.4 & 18.5 & 17.2 & 12.7 & 9.1 & 14.1 & 9.7 \\
$I_{\mathrm{YY}}$ & 129.2 & 163.4 & 118.9 & 116.0 & 116.2 & 161.9 & 105.3 \\
$I_{\mathrm{ZZ}}$ & 144.5 & 182.3 & 134.0 & 129.5 & 122.3 & 171.1 & 112.0 \\
Center of mass [cm] & 80.2 & 84.7 & 72.3 & 78.7 & 80.0 & 83.8 & 74.6 \\
Total mass [kg] & 82.2 & 98.5 & 65.2 & 75.5 & 73.4 & 90.9 & 72.5 \\
Height [cm] & 179.9 & 190.1 & 172.1 & 176.3 & 175.5 & 185.7 & 171.5 \\
\hline \hline
\end{tabular}


do not report data due to the small number of female astronauts. For several position of females we reported results in $[42,43]$.

Here we report only data for the "standing position" in Table 2 for females and in Table 3 for male. For more details the interested reader shall consult the abovementioned articles. In "standing position" the subject stands erect with the head oriented in the Frankfort plane and with arms hanging naturally at the sides as when measuring see Fig. 4. The 50\% and 95\% marks in the data of Hanavan [31] and NASA [38] mean that the corresponding percent of measured data is below the value reported in the table.

It is interesting to note that the typical astronaut is taller, by at least $8 \mathrm{~cm}$, but heavier, by at least $10 \mathrm{~kg}$, from the average Bulgarian male.

\section{Discussion}

Most of the results reported above, except those presented in Table 1, are obtained in the framework of the 16-segmental model proposed in [34]. It is clear that better the geometrical approximation of the real shapes of the segments of the body closer will be the mathematical results to those experimentally observed. In that respect on has at least two options for improving the modelling of the upper and lower extremities of the human body: instead of considering their cross sections to be ellipsis, one can consider realization of both the 16-segmental and of the 20-segmental models using cross-sections in the form of stadium or completely elliptic stadium, defining in this way what one can call model A and model B, respectively. The two models are explained in more details in Fig. 5 and 6.

In order to implement the recipe outlined in the Introduction, one needs the analytical properties of the 3D bodies presented in Fig. 6. Here we will present, due to the limitation of space, the analytical expression for the moments of inertia. The $I_{X X}$ moment of inertia with respect to a coordinate system centred at the bottom of the segment is

$$
\begin{aligned}
I_{X X} & =\frac{\rho}{720} L\left\{R _ { t 0 } \left\{3\left(4 R_{l 0}+R_{l 1}\right)\left[32 r_{t 0} R_{t 0}+3 \pi\left(4 r_{t 0}^{2}+R_{t 0}^{2}\right)\right]\right.\right. \\
& \left.+8\left(3 R_{l 0}+2 R_{l 1}\right)\left(3 \pi r_{t 0}+4 R_{t 0}\right) r_{t 1}+12 \pi\left(2 R_{l 0}+3 R_{l 1}\right) r_{t 1}^{2}\right\} \\
& +3 \pi\left\{3 \left[\left(4 r_{l 0}+r_{l 1}\right) r_{t 0}^{3}+\left(3 r_{l 0}+2 r_{l 1}\right) r_{t 0}^{2} r_{t 1}\right.\right. \\
& \left.+\left(2 r_{l 0}+3 r_{l 1}\right) r_{t 0} r_{t 1}^{2}+\left(r_{l 0}+4 r_{l 1}\right) r_{t 1}^{3}\right] \\
& \left.+4 L^{2}\left[r_{l 0}\left(2 r_{t 0}+3 r t 1\right)+3 r l 1\left(r_{t 0}+4 r_{t 1}\right)\right]\right\} \\
& +48\left\{\left(4 R_{l 0}+R_{l 1}\right) r_{t 0}^{3}+\left(3 R_{l 0}+2 R_{l 1}\right) r_{t 0}^{2} r_{t 1}\right. \\
& +\left(2 R_{l 0}+3 R_{l 1}\right) r_{t 0} r_{t 1}^{2}+\left(R_{l 0}+4 R_{l 1}\right) r_{t 1}^{3} \\
& \left.+L^{2}\left[R_{l 0}\left(2 r_{t 0}+3 r_{t 1}\right)+3 R_{l 1}\left(r_{t 0}+4 r_{t 1}\right)\right]\right\}
\end{aligned}
$$




$$
\begin{aligned}
& +\left[\left(3 R_{l 0}+2 R_{l 1}\right)\left(12 \pi r_{t 0}^{2}+64 r_{t 0} R_{t 0}+9 \pi R_{t 0}^{2}\right)\right. \\
& +8\left(2 R_{l 0}+3 R_{l 1}\right)\left(3 \pi r_{t 0}+8 R_{t 0}\right) r_{t 1} \\
& \left.+36 \pi\left(R_{l 0}+4 R_{l 1}\right) r_{t 1}^{2}\right] R_{t 1}+\left[\left(2 R_{l 0}+3 R_{l 1}\right)\left(32 r_{t 0}+9 \pi R_{t 0}\right)\right. \\
& \left.+96\left(R_{l 0}+4 R_{l 1}\right) r_{t 1}\right] R_{t 1}^{2}+9 \pi\left(R_{l 0}+4 R_{l 1}\right) R_{t 1}^{3} \\
& \left.+12 L^{2} \pi\left[R_{l 0}\left(2 R_{t 0}+3 R_{t 1}\right)+3 R_{l 1}\left(R_{t 0}+4 R_{t 1}\right)\right]\right\}
\end{aligned}
$$

The expression above are for $I_{X X}$ in a coordinate frame centred at the middle of the lower bounding surface, i.e., at the point $(x=0, y=0, z=0)$. In order to determine the principal moment of inertia $I_{X X}^{(C M)}$, we have to use the Steiner's theorem. One can write expression for the position of the centre of mass $C M$, as well as for $I_{Y Y}, I_{Z Z}, I_{Y Y}^{(C M)}, I_{Z Z}^{(C M)}$. Since, as for $I_{X X}$, the corresponding expressions are quite lengthy and cumbersome, we will avoid doing that here.

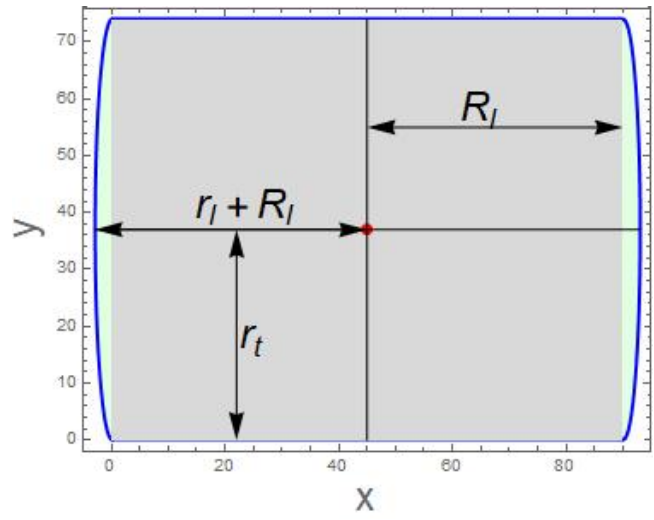

(a) The cross-section $\mathcal{S}_{A}$ of the upper and lower arm in model $A$. It consists of a rectangular $\mathcal{R}$ (light grey color) with horizontal length $2 R_{l}$ and vertical one $2 r_{t}$. On the left and the right-hand side of $\mathrm{R}$ one has two semiellipsis $\mathcal{E}_{l}$ and $\mathcal{E}_{r}$ (the light blue color) with semi-axis $r_{l}$ and $r_{t}$. Thus, one has $\mathcal{S}_{A}=$ $\mathcal{R} \bigcup E_{r}$, where $E_{r}=\mathcal{E}_{l} \cup \mathcal{E}_{r}$. Obviously, $E_{r}$ is an ellipse with semi-axis $r_{l}$ and $r_{t}$.

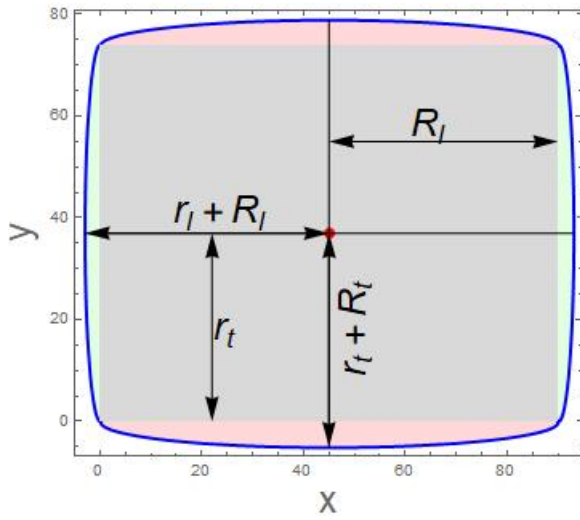

(b) The cross-section $\mathcal{S}_{B}$ of the upper and lower arm in model $B$. The cross section is similar to that one in model $A$ with the addition of two semi-ellipsis $\mathcal{E}_{t}$ and $\mathcal{E}_{b}$ (the light red color) on the top and the bottom of $\mathcal{R}$. The semi-ellipsis are with major semi-axis $R_{l}$ and minor semi-axis $R_{t}$. Obviously $E_{R}=\mathcal{E}_{t} \cup \mathcal{E}_{b}$ is an ellipse with semi-axis $R_{l}$ and $R_{t}$. Thus, one has $\mathcal{S}_{B}=\mathcal{R} \bigcup E_{r} \bigcup E_{R}$.

Fig. 5: The cross-sections of the upper and lower arm in models $A$ and $B$. Let us note that here we have shown some specific realization with values of the parameters pertinent for the upper arm. 


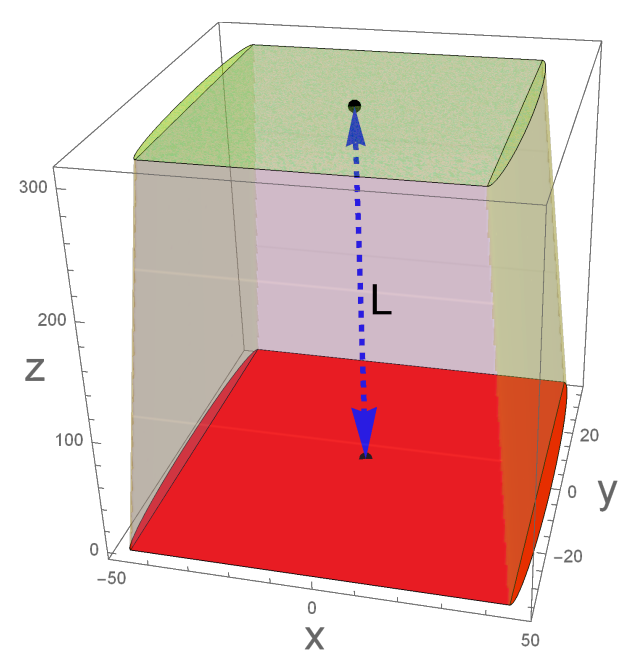

(a) Model $A$ : In this $3 D$ body at the bottom with $z=0$ one thinks of a cross section $\mathcal{S}_{A, 0}$ characterized with lengths $R_{l, 0}, r_{l, 0}, r_{t, 0}$ and on the top, for $z=L$, of a cross section $\mathcal{S}_{B, 1}$, with lengths $R_{l, 1}, r_{l, 1}, r_{t, 1}$. The height of the body, the distance between $\mathcal{S}_{B, 0}$ and $\mathcal{S}_{B, 1}$ is $L$. Since the midpoints of all cross sections lie on the $z$ axes, see Fig. 5(a), the $3 D$ body depicted above is normally termed right elliptic stadium solid (RESS).

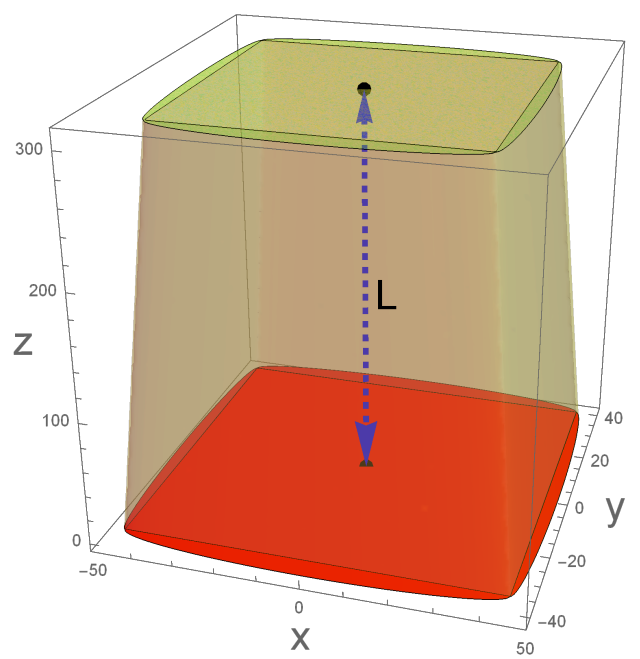

(b) Model $B$ : In this $3 D$ body at the bottom with $z=0$ one thinks of a cross section $\mathcal{S}_{B, 0}$ characterized with lengths $R_{l, 0}, R_{t, 0}, r_{l, 0}, r_{t, 0}$ and on the top, for $z=$ $L$, of a cross section $\mathcal{S}_{B, 1}$, with lengths $R_{l, 1}, R_{t, 1}, r_{l, 1}, r_{t, 1}$. The height of the body, the distance between $\mathcal{S}_{B, 0}$ and $\mathcal{S}_{B, 1}$ is $L$. As in model $A$ the midpoints of all cross sections lie on the $z$ axes, see Fig. 5(b). The $3 D$ body shown here will be termed right fully elliptic stadium solid (RFESS).

Fig. 6: A general view of the body with which we model, say, the upper and lower arm of the upper limb in models $A$ and $B$. In the figures the data for the upper arm are used. The dimension along the $z$ axes in vertical direction is not in scale with the ones along $x$ and $y$ in order to achieve better visibility.

\section{CONCLUSIONS}

All the results reported in the current article can be generalized by using models A and $\mathrm{B}$. It remains to be seen to which extend they will improve the agreement with the experimentally obtained data. Next, up to now we reported results for the average Bulgarian male and female. In order to implement fully model B one needs additional anthropometric data that are not available from [35]. They have been performed and one shall also analyse them properly in order to have the needed statistical data. Next, it is clear the all the studies reported up to now can be implemented to study the massinertial characteristics for one specific individual, not just for the "average" person. 
Obviously, in order to achieve that one only needs the set of anthropometric data for the individual of interest to be measured. Finally, let us note that the procedure we have outlined and followed is, by no means, specific for the Bulgarian population. It can be done for any group of people for which the corresponding data are known. We suppose that the proposed models can find proper applications in medicine (orthopaedics and traumatology), rehabilitation robotics, computer simulations, sports and areas such as ergonomics, forensics and more.

\section{ACKNOWLEDGEMENTS}

The authors gratefully acknowledge the financial support via contract DN 07/5 with Bulgarian National Science Fund.

\section{REFERENCES}

[1] G.F. Harris, P.A. Smith (1996) "Human Motion Analysis: Current Applications and Future Directions". IEEE Press Text, New York.

[2] W. Braune, O. Fischer (1987) "The Human Gait". Springer-Verlag, Berlin.

[3] D. Winter (1991) "The Biomechanics and Motor Control of Human Gait: Normal, Elderly and Pathological" (2-nd. ed.). Waterloo Biomechanics Waterloo, Canada.

[4] V.M. ZATSIORSKY (2002) "Kinetics of human motion". Human Kinetics, Champaign, IL, USA.

[5] D.A. Winter (2009) "Biomechanics and Motor Control of Human Movement". Wiley, New Jersey, USA.

[6] I.P. Herman (2016) "Physics of the Human Body". Springer Verlag, Berlin.

[7] W. Thornton, F. Bonato (2017) "The Human Body and Weightlessness. Operational Effects, Problems and Countermeasures". Springer Verlag, Berlin.

[8] J.F. Lehmann, S.M. Condon, B.J. De Lateur, R. PriCe (1986) Gait abnormalities in peroneal nerve paralysis and their corrections by orthoses: a biomechanical study. Archives of Physical Medicine and Rehabilitation 67 380-386.

[9] J.F. Lehmann, S.M. Condon, R. Price, B.J DE LATeur (1987) Gait abnormalities in hemiplegia: their correction by ankle-foot orthoses. Archives of Physical Medicine and Rehabilitation 68 763-771.

[10] S. Olney, M. GRifFin, T. Monga, I. McBRide (1991) Work and power in gait of stroke patients Archives of Physical Medicine and Rehabilitation 72 309-314.

[11] M. Collopy, M. Murray, G. Gardner, R. Di Unio, D. Gore (1977) Kinesiologic measurements of functional performance before and after geometric total knee replacement: one-year follow-up of twenty cases. Clinical Orthopaedics and Related Research 126 196-202.

[12] N. Rittman, D. Kettelkamp, P. Pryor, G. Schwarzkopf, B. Hillbarry (1981) Analysis of patterns of knee motion walking for four types of total knee implants. Clinical Orthopaedics and Related Research 155 111-117. 
[13] M.W. Whittle (1991) “Gait Analysis: An Introduction”. Butterworth-Heinemann Ltd Halley Court, Jordan Hill, Oxford, UK.

[14] P.M. MCGinnis (2013) "Biomechanics of sport and exercise", 3rd ed. Human Kinetics, Champaign, IL, USA.

[15] A. BlazeViCH (2007) "Sports biomechanics. The basics: optimising human performance". $A \& C$ Black Publishers Ltd, London, UK.

[16] Y. Hong, R. BARTlett, eds. (2008) "Routledge Handbook of Biomechanics and Human Movement Science”. Routledge, USA.

[17] V. ZATSIORSKY (2000) "Biomechanics in Sport: Performance Enhancement and Injury Prevention". Wiley-Blackwell, USA.

[18] H. LeE, S. Sullivan, A. Schneiders (2013) The use of the dual-task paradigm in detecting gait performance deficits following a sports-related concussion: a systematic review and meta-analysis. Journal of Science and Medicine in Sport 16 2-7.

[19] J. Kieser, M. TAYLOR, D. CARR, eds. (2012) "Forensic Biomechanics". Wiley, USA.

[20] F. DARREN, F. HAROLD, eds. (2016) "Forensic biomechanics and human injury: criminal and civil applications: an engineering approach". CRC Press, USA.

[21] S. KUMAR (2007) "Biomechanics in Ergonomics", 2 ed.. CRC Press, USA.

[22] M. B. Popović (2013) “Biomechanics and Robotics”. CRC Press, USA.

[23] W.T. DEMPSTER (1955) "Space Requirements of the Seated operator. WADC Technical Report ". Wright-Patterson Air Force Base, Ohio, USA pp. 55-159.

[24] C.E. Clauser, J.T. McConville, J.W. Young (1969) "Weight, Volume, and Center of Mass of Segments of the Human Body. Technical Report AMRL-TR-69-70". Wright-Patterson Air Force Base, Ohio, USA.

[25] R.F. Chandler, C.E. Clauser, J.T. McConville, H.M Reynolds, J.W. Young (1975) "Investigation of Inertial Properties of the Human Body. Technical Report AMRL-TR-74-137”. Wright-Patterson Air Force Base, Ohio, USA.

[26] R. Drillis, R. Contini (1966) "Body Segment Parameters. Techn. Report No 1166.03, PB 174945". School of Engineering and Science, New York University. Aerospace Medical Research Laboratory, Wright-Patterson Air Force Base, Ohio, USA.

[27] V.M. Zatsiorsky, V.N. Seluyanov (1983) "The Mass and Inertia Characteristics of the Main Segments of the Human Body.”. In: “Biomechanics VIII-B”, H. Matsui, K. Kobayashi K. (eds.), Champaign, IL: Human Kinetics, pp. 1152-1159.

[28] V.M. Zatsiorsky, V.N. Seluyanov (1985) "Estimation of the Mass and Inertia Characteristics of the Human Body by Means of the Best Predictive Regression Equations.”. In: “Biomechanics IX-B”, D. Winter, R. Norman, R. Wells, K. Hayes, A. Patla (eds.), Champaign, IL: Human Kinetics, pp. 233-239.

[29] M. Munigiole, P.E. Martin (1990) Estimating Segment Inertial Properties: Comparison of Magnetic Resonance Imaging with Existing Methods. Journal of Biomechanics 117 1039-1046; DOI: https://doi.org/10.1016/0021-9290(90)90319-X 
[30] C. WEI, R.K. JENSEN (1995) The application of segment axial density profiles to a human body inertia model. Journal of Biomechanics 28 103-108; DOI: https://doi.org/10.1016/0021-9290(95)80012-3.

[31] E.P. HANAVAN (1964) "A mathematical model of the human body. AMRL-TR-64102”. Aerospace Medical Research Laboratory, Wright-Patterson Air Force Base, Ohio, USA.

[32] M.R. YEADON (1990) The simulation of aerial movement-II. A mathematical inertia model of the human body. Journal of Biomechanics 23 67-74; DOI: https://doi.org/10.1016/0021-9290(90)90370-I.

[33] Y.H. KwON 3D Motion Analysis. Web site: http://kwon3d.com_103946.

[34] G. Nikolova, Y. Toshev (2007) Estimation of Male and Female Body Segment Parameters of the Bulgarian Population Using a 16-segmental Mathematical Model. Journal of Biomechanics 40 3700-3707; DOI: https://doi.org/10.1016/j.jbiomech.2007.06.016.

[35] Y. Yordanov, A. Nacheva, S. Tornjova, N. Kondova, B. Dimitrova, D. Topalova (2006) "Anthropology of the Bulgarian Population at the end of the 20th Century (30-40 Years Old Persons)”. Prof. M. Drinov Publishing House, Sofia, Bulgaria.

[36] G. Nikolova, M.S. Tsveov, D.M. Dantchev (2019) A Mathematical Model of the Human Thigh and its Connection with the Torso. AIP Conference Proceedings 2164 080006; DOI: https://doi.org/10.1063/1.5130829.

[37] G. Shan, C. BoHn (2003) Anthropometrical Data and Coefficients of Regression Related to Gender and Race. Applied Ergonomics 34 327-337; DOI: https://doi.org/10.1016/S0003-6870(03)00040-1.

[38] NASA-STD-3000 "The mansystem integration standards, Anthropometry and biomechanics.”. https://msis.jsc.nasa.gov/sections/section03.htm\#3.2.1.

[39] G.S. Nikolova, V.K. Kotev, D.M. DantCheV (2017) "CAD modelling of human body for robotics applications.'. In Proceedings of International Conference on Control, Artificial Intelligence, Robotics \& Optimization, IEEE Computer Society Conference Publishing Services (CPS), Prague, Czech Republic, 20-22 May 2007.

[40] V.K. Kotev, G.S. Nikolova, D.M. Dantchev (2017) "Determination of massinertial characteristics of the human body in basic body positions: computer and mathematical modelling.”. In: IFMBE Proceedings 65, EMBEC \& NBC 2017, Tampere, Finland, 11-15 June, 2017, Springer Nature, Singapore Pte Ltd.

[41] G.S. Nikolova, V.K. Kotev, D.M. Dantchev (2018) CAD design of human male body for massinertial characteristics studies. MATEC Web of Conferences 14504006 ; DOI: https://doi.org/10.1051/matecconf/201814504006.

[42] V.K. Kotev, G.S. Nikolova, D.M. DantCheV (2017) "Computer and mathematical modelling of the female human body: determination of mass-inertial characteristics in basic body positions.". In: Proceedings of the 7th International Conference on Simulation and Modeling Methodologies, Technologies and Applications (SIMULTECH 2017), SCITEPRESS, Madrid, Spain, 26-28 July 2017. 
[43] G.S. Nikolova, V.K. Kotev, D.M. Dantchev (2018) Results for females massinertial parameters in basic body positions for space exploration as classified by NASA AIP Conference Proceedings 2075 170006; DOI: https://doi.org/10.1063/1.5091371.

[44] J.W. Young, R.F. Chandler, C.C. Snow, K.M. Robinette, G.F. Zehner, M.S. LOFBERG (1983) "Anthropometric and mass distribution characteristics of the adult female.”. Technical Report FAA-AM-83-16, Oklahoma City, USA.

[45] S. Abraham, C.L. Johnson, M. NAJjar (1979) "Weight and height of adults 18-74 Years of Age: United States 1971-74, DHEW Publication No (PHS) 79-1659”. Hyattsville, Md., USA.

[46] W.R. SAntschi (1963) "Moments of Inertia and Centers of Gravity of the Living Human Body. AMRL TR 63-36.” Wright-Patterson Air Force Base, Ohio, USA. 